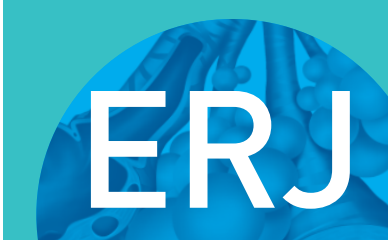

open research
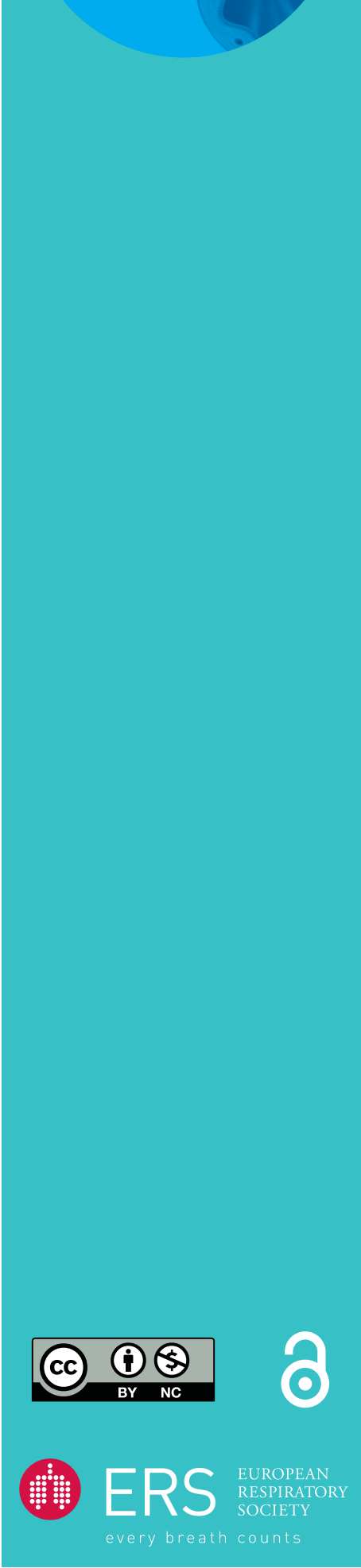

\section{Metformin use and post-exposure incident tuberculosis: a nationwide tuberculosis-contact cohort study in Taiwan}

\section{To the Editor:}

Patients with diabetes mellitus are susceptible to active tuberculosis (TB) and latent TB infection (LTBI) [1-3]. Close contact with patients with infectious $\mathrm{TB}$ is associated with an increased risk of having coprevalent TB or developing incident TB $[4,5]$. However, information on the burden of incident TB in contacts with underlying diabetes is limited. Considering post-exposure $\mathrm{TB}$ in patients with diabetes, metformin has demonstrated anti-TB effects in preclinical studies and association with a low LTBI prevalence in a cross-sectional survey $[6,7]$. Despite metformin's wide use and correlation with a lower TB risk in patients with type 2 diabetes (T2D) [8], the impact of metformin use during TB exposure on subsequent $\mathrm{TB}$ risk has not been thoroughly investigated. We aimed to obtain the rate of post-exposure incident $\mathrm{TB}$, but not coprevalent TB, in T2D patients and evaluate whether TB risk can be modified by metformin use during the TB-exposure period.

This retrospective cohort study in TB contacts used the National Health Insurance Research Database (with approval from Taipei City Hospital: TCHIRB-10704109-W), in a manner as previously used by YEN et al. [9]. This database includes longitudinal claims data of $>23$ million beneficiaries in Taiwan and has contributed to numerous validated reports $[4,8]$. We identified TB contacts by diagnostic International Classification of Diseases, ninth revision, clinical modification (ICD-9-CM) code of V01.1 plus chest radiography orders in outpatient records from 2008 to 2014, when TB-contact investigation is mandatory in Taiwan [4]. The index date was the date of coding of V01.1. To minimise confounding variables, we excluded subjects with a prior TB history, preventive therapy for LTBI, type 1 diabetes and those with chronic kidney disease, in who metformin is contradicted [8].

We defined T2D patients by the presence of 250.x0 and 250.x2 ICD-9 codes (one inpatient or more than three outpatient records in the 6 months before and after the index date) [8]. Using the defined daily dose (DDD) of metformin [8], i.e. $2000 \mathrm{mg}$, we defined a T2D subject as having metformin use during TB exposure (T2D-useMet) if the cumulative DDD of metformin was $>30$ within 6 month before the index date; the others were T2D without metformin (T2D-nonMet). The outcome, after excluding subjects with coprevalent $\mathrm{TB}$ occurring within 6 months, was post-exposure incident TB developing after the initial 6 months [5]. We identified TB by codes $010-018$ plus prescription of anti-TB drugs in all subjects until December 2016, death or 3 years [8]. We recorded potential confounders including diabetes severity as indicated by the adapted diabetes complication severity index (aDCSI) score (table 1) [8]. We used SAS (version 9.4; SAS Institute, Cary, NC, USA) for data extraction and analysis, and Cox regression analysis to assess predictors for incident TB.

We identified 234373 adult TB contacts aged $>20$ years during 2008-2014. We excluded 3308 (1.4\%) participants with prior TB, $1414(0.6 \%)$ with coprevalent TB within 6 months after the index dates and

@ERSpublications

This TB contact cohort study showed that the risk of incident TB, not coprevalent TB, was highest in the diabetes group without metformin use during TB exposure, followed by the nondiabetes population, and was lowest in the diabetes group with metformin use https://bit.ly/ 3fpJyF0

Cite this article as: Pan S-W, Feng J-Y, Yen Y-F, et al. Metformin use and post-exposure incident tuberculosis: a nationwide tuberculosis-contact cohort study in Taiwan. ERJ Open Res 2020; 6: 00050-2020 [https://doi.org/10.1183/23120541.00050-2020]. 
TABLE 1 Demographic data of the whole cohort and analysis of the risk factors for post-exposure incident tuberculosis (TB) in patients with type 2 diabetes (T2D)

\begin{tabular}{|c|c|c|c|c|c|c|c|c|}
\hline \multirow[t]{3}{*}{ Variables } & \multirow[t]{3}{*}{ TB contact cohort } & \multirow[t]{3}{*}{ Nondiabetes participants } & \multicolumn{2}{|c|}{ T2D participants } & \multicolumn{4}{|c|}{ Cox analysis of risk factors for TB in T2D population } \\
\hline & & & \multirow[t]{2}{*}{ Non-metformin } & \multirow[t]{2}{*}{ Metformin use } & \multicolumn{2}{|c|}{ Univariate analysis } & \multicolumn{2}{|c|}{ Multivariate analysis } \\
\hline & & & & & HR $(95 \% \mathrm{Cl})$ & p-value & HR $(95 \% \mathrm{Cl})$ & p-value \\
\hline Subjects $\mathrm{n}$ & 205797 & 185189 & 9851 & 10757 & & & & \\
\hline Age years & $46.24 \pm 15.17$ & $44.90 \pm 14.44$ & $59.10 \pm 16.74$ & $57.49 \pm 15.70$ & $1.01(1.00-1.02)$ & 0.088 & $1.00(0.99-1.02)$ & 0.455 \\
\hline Male sex & 106301 (51.65\%) & $94523(51.04 \%)$ & 5719 (58.06\%) & 6059 (56.33\%) & $1.70(1.19-2.43)$ & 0.004 & $1.71(1.18-2.47)$ & 0.005 \\
\hline \multicolumn{9}{|l|}{ Income level } \\
\hline Low & 39865 (19.37\%) & 34088 (18.41\%) & 2970 (30.15\%) & 2807 (26.09\%) & 1.00 & & 1.00 & \\
\hline Intermediate & $112053(54.45 \%)$ & 101464 (54.79\%) & $4832(49.05 \%)$ & $5757(53.52 \%)$ & $0.84(0.58-1.22)$ & 0.372 & $1.01(0.68-1.51)$ & 0.942 \\
\hline High & 53879 (26.18\%) & $49637(26.80 \%)$ & $2049(20.80 \%)$ & $2193(20.39 \%)$ & $0.69(0.42-1.13)$ & 0.141 & $0.89(0.52-1.52)$ & 0.669 \\
\hline \multicolumn{9}{|l|}{ Urbanisation } \\
\hline Urban & 114124 (55.45\%) & 103798 (56.05\%) & 4951 (50.26\%) & 5375 (49.97\%) & 1.00 & & 1.00 & \\
\hline Suburban & 70748 (34.38\%) & $63307(34.19 \%)$ & $3529(35.82 \%)$ & $3912(36.37 \%)$ & $1.16(0.80-1.69)$ & 0.441 & $1.11(0.75-1.63)$ & 0.601 \\
\hline Rural & $20925(10.17 \%)$ & $18084(9.77 \%)$ & $1371(13.92 \%)$ & $1470(13.67 \%)$ & $1.99(1.30-3.06)$ & 0.002 & $1.83(1.17-2.88)$ & 0.008 \\
\hline \multicolumn{9}{|l|}{ Comorbidities } \\
\hline COPD or asthma & 24763 (12.03\%) & 19316 (10.43\%) & 3039 (30.85\%) & 2408 (22.39\%) & $1.49(1.05-2.12)$ & 0.027 & $1.24(0.85-1.80)$ & 0.261 \\
\hline Malignancy & 6712 (3.26\%) & 5080 (2.74\%) & $869(8.82 \%)$ & $763(7.09 \%)$ & $1.19(0.66-2.15)$ & 0.565 & $1.03(0.57-1.89)$ & 0.913 \\
\hline Liver cirrhosis & 1966 (0.96\%) & 1281 (0.69\%) & $396(4.02 \%)$ & $289(2.69 \%)$ & $2.81(1.52-5.20)$ & 0.001 & $2.00(1.02-3.92)$ & 0.042 \\
\hline Alcoholism & $1498(0.73 \%)$ & $1151(0.62 \%)$ & $214(2.17 \%)$ & $133(1.24 \%)$ & $3.24(1.52-6.94)$ & 0.002 & $1.94(0.84-4.47)$ & 0.119 \\
\hline Autoimmune disease & $5508(2.68 \%)$ & $4450(2.40 \%)$ & $532(5.40 \%)$ & 526 (4.89\%) & $0.80(0.35-1.81)$ & $0.594^{\#}$ & $0.72(0.32-1.65)$ & $0.440^{\#}$ \\
\hline AIDS $^{\#}$ & 234 & 164 (0.09\%) & $8(0.08 \%)$ & 4 & & & & \\
\hline Organ Transplant ${ }^{\#}$ & & $48(0.03 \%)$ & $10(0.10 \%)$ & & & & & \\
\hline Steroid use" & $1408(0.68 \%)$ & $1093(0.59 \%)$ & $172(1.75 \%)$ & $143(1.33 \%)$ & $2.25(0.83-6.09)$ & 0.110 & $1.88(0.69-5.12)$ & 0.220 \\
\hline Statin use $e^{\pi}$ & $9653(4.69 \%)$ & $4256(2.30 \%)$ & 1639 (16.64\%) & 3758 (34.94\%) & $0.66(0.44-1.00)$ & 0.049 & $0.77(0.51-1.18)$ & 0.236 \\
\hline Insulin use" & $1747(0.85 \%)$ & $5(0.00 \%)$ & $808(8.20 \%)$ & 934 (8.68\%) & $1.16(0.66-2.05)$ & 0.612 & $1.11(0.62-2.00)$ & 0.723 \\
\hline aDCSI score & $0.64 \pm 1.24$ & $0.46 \pm 0.96$ & $2.27 \pm 2.04$ & $2.30 \pm 1.96$ & $1.06(0.98-1.15)$ & 0.153 & $1.03(0.94-1.12)$ & 0.542 \\
\hline Follow-up duration years & $2.93 \pm 0.35$ & $2.94 \pm 0.30$ & $2.71 \pm 0.68$ & $2.83 \pm 0.51$ & & & & \\
\hline Incident TB events & $912(0.44 \%)$ & $771(0.42 \%)$ & $82(0.83 \%)$ & $59(0.55 \%)$ & & & & \\
\hline \multicolumn{9}{|l|}{ T2D status on TB exposur } \\
\hline T2D non-metformin & $9851(4.78 \%)$ & & & & 1.00 & & 1.00 & \\
\hline T2D metformin use & $10757(5.22 \%)$ & & & & $0.63(0.45-0.88)$ & 0.007 & $0.69(0.49-0.98)$ & 0.036 \\
\hline
\end{tabular}

Data are presented as $n(\%)$ or mean \pm SD, unless otherwise stated. HR: hazard ratio; aDCSI: adapted diabetes complication severity index. \#: because the numbers of AIDS and organ transplant patients were too small to be publicly disclosed in the table, the two factors were combined into autoimmune disease for the univariate and multivariate Cox regression analyses. ": use of a comedication during TB exposure period is defined as taking a drug for $>30$ cumulative defined daily doses within 6 months before the index date. 
$6161(2.6 \%)$ receiving isoniazid preventive therapy for LTBI. After excluding subjects with chronic kidney disease and/or type 1 diabetes $(n=5172)$ and T2D developed during 3-year follow-up $(n=12521)$, we finally included 205797 participants for the analysis: 185189 (90\%) nondiabetes, 9851 (4.8\%) T2D-nonMet and 10757 (5.2\%) T2D-useMet subjects. Compared with the nondiabetes group, the T2D groups were older and had more comorbidities and comedications, including statins. After a mean \pm SD follow-up of $2.93 \pm 0.35$ years, we observed 912 (0.44\%) incident TB events: $771(0.42 \%), 82(0.83 \%)$ and 59 $(0.55 \%)$ in the nondiabetes, T2D-nonMet and T2D-useMet groups $(\mathrm{p}<0.001)$, respectively. The corresponding incidences of TB were 1.4, 3.1 and 1.9 cases per 1000 person-years in the three groups (table 1). The Kaplan-Meier curves of probabilities of incident TB after the initial post-exposure 6 months for the three groups were significant different (log-rank $\mathrm{p}<0.001)$.

In the $\mathrm{T} 2 \mathrm{D}$ population, metformin use during $\mathrm{TB}$ exposure significantly predicted incident $\mathrm{TB}$ in univariate analysis $(p=0.007)$. Notably, T2D-useMet was negatively associated with TB after adjustment by cofactors with univariate $\mathrm{p}<0.05$ (adjusted hazard ratio (aHR) $0.69,95 \%$ CI $0.49-0.98 ; \mathrm{p}=0.037$ ). In the fully adjusted model, T2D-useMet remained correlated with lower risk for TB development (aHR 0.69, 95\% CI 0.49-0.98; p=0.036) (table 1). Furthermore, compared with T2D-nonMet subjects, T2D-useMet subjects receiving a high dose of metformin had further declined risks of TB: aHR 0.87 (95\% CI 0.56-1.37, $\mathrm{p}=0.558)$ in the subgroup receiving 30-90 DDD and 0.59 (95\% CI $0.39-0.90, \mathrm{p}=0.013)$ in that taking $>90$ DDD within 6 months before the index date. While incorporating nondiabetes data into the analysis, we found that TB risk was highest in the T2D-nonMet group (aHR 1.41, 95\% CI 1.01-1.97; $\mathrm{p}=0.045$ ), followed by the nondiabetes (aHR 1.15, 95\% CI 0.86-1.52; p=0.342), and was lowest in T2D-useMet (reference) groups after adjusting for age, sex, income, residence, all comorbidities, steroid and insulin use (all factors with univariate $\mathrm{p}<0.05$ in the whole-population analysis; statins not included due to $\mathrm{p}=0.917$ in the univariate analysis).

In summary, our diabetes subjects with metformin use during TB exposure had a $31 \%$ lower risk of incident TB than those without and a similar risk to the controls without diabetes. In another TB contact study (5846 matched trios), LEE et al. [10] reported that, compared with diabetes without metformin, TB risk was $27 \%$ lower in matched metformin users taking $\geqslant 90$ DDD within 1 year (aHR 0.73) and lowest in healthy contacts without any comorbidities (aHR 0.42). We think that their overtly healthy controls and accounting for coprevalent TB as the outcome may explain the deviation in their results from ours. Our study excluded coprevalent TB and found only $0.44 \%$ of contacts developed incident TB within 3 years. This finding is consistent with the result of the Amsterdam study: 0.39\% incident TB events within 5 years [3]. Importantly, we present a dose-response association between metformin and incident TB. Our robust result suggests that metformin may modify the risk of TB disease in patients with diabetes who have been exposed to TB patients [7].

Regarding limitations, we lacked data on smoking, exposure types or LTBI status of the contacts and the sputum smear status of the index cases [5]. Second, because metformin might be used before TB exposure throughout the following years, whether metformin acts to prevent initial infection or reactivation from LTBI remains unknown.

In conclusion, we found, in patients with diabetes taking metformin, a decreased risk of post-exposure incident $\mathrm{TB}$, in a low level similar to that in subjects without diabetes. These results may provide rationales for researching metformin's potential in preventive therapy after TB exposure.

Sheng-Wei Pan $\circledast^{1,2,3}$, Jia-Yih Feng ${ }^{1,2,4}$, Yung-Feng Yen ${ }^{2,3,4,5}$, Fan-Yi Chuang ${ }^{1}$, Hsiang-Shi Shen ${ }^{1}$, Vincent Yi-Fong Su ${ }^{2,6,7}$, Pei-Hung Chuang ${ }^{8}$ Yu-Jiun Chan ${ }^{3,9,10}$ and Wei-Juin Su ${ }^{1,2}$

${ }^{1}$ Dept of Chest Medicine, Taipei Veterans General Hospital, Taipei, Taiwan. ${ }^{2}$ Faculty of Medicine, National Yang-Ming University, Taipei, Taiwan. ${ }^{3}$ Institute of Public Health, National Yang-Ming University, Taipei, Taiwan. ${ }^{4}$ Institute of Clinical Medicine, National Yang-Ming University, Taipei, Taiwan. ${ }^{5}$ Section of Infectious Diseases, Taipei City Hospital, Taipei, Taiwan. ${ }^{6}$ Dept of Health Care Management, National Taipei University of Nursing and Health Sciences, Taipei, Taiwan. ${ }^{7}$ Dept of Internal Medicine, Taipei City Hospital, Taipei, Taiwan. ${ }^{8}$ Taipei Association of Health and Welfare Data Science, Taiwan. ${ }^{9}$ Division of Infectious Diseases, Dept of Medicine, Taipei Veterans General Hospital, Taipei, Taiwan. ${ }^{10}$ Division of Microbiology, Dept of Pathology and Laboratory Medicine, Taipei Veterans General Hospital, Taipei, Taiwan.

Correspondence: Wei-Juin Su, Dept of Chest Medicine, Taipei Veterans General Hospital, No. 201, Sec. 2, Shih-Pai Rd., Taipei 11217, Taiwan. E-mail: wjsu.mail@gmail.com

Received: 30 Jan 2020 | Accepted after revision: 28 April 2020 
Author contributions: S-W. Pan, J-Y. Feng, Y-J. Chan and V.Y-F. Su conceived and designed this study. S-W. Pan, Y-F. Yen and P-H. Chuang acquired and analysed the data, and had full access to all of the data. All authors contributed to the interpretation of the results of this study. S-W. Pan, J-Y. Feng, Y-J. Chan and W-J. Su contributed to drafting the manuscript. Y-F. Yen and W-J. Su are the supervisors of the paper and responsible for the integrity of the work as a whole.

Support statement: S-W. Pan is supported by grants from Taipei Veterans General Hospital (grant numbers V108B-039 and V109C-053) and the Ministry of Science and Technology, Taiwan (MOST 107-2314-B-075 -057 and 108-2314-B-075-001). Funding information for this article has been deposited with the Crossref Funder Registry.

Conflict of interest: None declared.

\section{References}

1 Jeon CY, Murray MB. Diabetes mellitus increases the risk of active tuberculosis: a systematic review of 13 observational studies. PLoS Med 2008; 5: e152.

2 Lee MR, Huang YP, Kuo YT, et al. Diabetes mellitus and latent tuberculosis infection: a systematic review and metaanalysis. Clin Infect Dis 2017; 64: 719-727.

3 Jackson C, Southern J, Lalvani A, et al. Diabetes mellitus and latent tuberculosis infection: baseline analysis of a large UK cohort. Thorax 2019; 74: 91-94.

4 Pan SW, Yen YF, Feng JY, et al. The risk of depressive disorder among contacts of tuberculosis patients in a TB-endemic area: a population-based cohort study. Medicine (Baltimore) 2015; 94: e1870.

5 Sloot R, van der Loeff MF S, Kouw PM, et al. Risk of tuberculosis after recent exposure. A 10-year follow-up study of contacts in Amsterdam. Am J Respir Crit Care Med 2014; 190: 1044-1052.

6 Singhal A, Jie L, Kumar P, et al. Metformin as adjunct antituberculosis therapy. Sci Transl Med 2014; 6: 263 ra159.

7 Magee MJ, Salindri AD, Kornfeld $\mathrm{H}$, et al. Reduced prevalence of latent tuberculosis infection in diabetes patients using metformin and statins. Eur Respir J 2019; 53: 1801695.

8 Pan SW, Yen YF, Kou YR, et al. The risk of TB in patients with type 2 diabetes initiating metformin $v s$ sulfonylurea treatment. Chest 2018; 153: 1347-1357.

9 Yen YF, Feng JY, Yi-Fong Su V, et al. Human immunodeficiency virus infection increases the risk of incident uveitis among people living with HIV/AIDS. J Acquir Immune Defic Syndr 2018; 79: 149-157.

10 Lee MC, Lee $\mathrm{CH}$, Lee MR, et al. Impact of metformin use among tuberculosis close contacts with diabetes mellitus in a nationwide cohort study. BMC Infect Dis 2019; 19: 936. 